\title{
Addressing Beta-lactam Allergy: A Time for action
}

\author{
Elizabeth Philips ${ }^{1}$, Pascal Demoly ${ }^{2}$, and Maria Torres ${ }^{3}$ \\ ${ }^{1}$ Vanderbilt University Department of Medicine \\ ${ }^{2}$ Montpellier Universite d'Excellence \\ ${ }^{3}$ Universidad de Malaga Facultad de Medicina
}

September 25, 2021

\section{Addressing Beta-lactam Allergy: A Time for action}

Elizabeth J. Phillips, MD, FIDSA, FAAAAI, Pascal Demoly, MD, PhD, Maria J Torres, MD, PhD

${ }^{1}$ Department of Medicine, Center for Drug Safety and Immunology, Vanderbilt University Medical Center, Nashville Tennessee USA, ${ }^{2}$ Institute for Immunology \& Infectious Diseases, Murdoch University, Murdoch Australia, ${ }^{3}$ Division of Allergy, Department of Pulmonology, University Hospital of Montepellier, and IDESP, Univ. Montpellier - Inserm, Montpellier France, ${ }^{4}$ Allergy Unit, Hospital Regional Universitario de MalagaIBIMA-BIONAND-ARADyAL, and Departmento de Medicina, Universidad de Malaga, Malaga, Spain

Correspondence:

Elizabeth J. Phillips, MD, FIDSA, FAAAAI

Center for Drug Safety and Immunology

Vanderbilt University Medical Center

$1161-21^{\text {st }}$ Avenue South

Nashville, TN 37232

(615) 322-9174 (tel)

Elizabeth.j.phillips@vumc.org

It is now 93 years since the discovery of penicillins, and over 75 years since the first use of penicillin. We have entered yet another wave of challenges plagued with antibiotic resistance accelerating at a rate that well exceeds that of new antibiotic development. In the face of these uphill battles, $8-15 \%$ of a global population who has had access to care is labeled as penicillin allergic. ${ }^{1}$ In the United States (US) there are at maximum 6000 specialists who practice allergy out of a total of 700,000 practicing physicians, and not all allergists are proficient in and practice drug allergy. Conservatively out of 30,000,000 who are labeled as penicillin allergic at any one time in the US, this would mean that each allergist would need to delabel a minimum of 6000 patients. In Europe and the United Kingdom, the figures are proportionately identical, with some differences between countries. Even if all patients had equal access to care, this type of scalability remains impossible. This overwhelming burden that threatens to negatively impact healthcare through delays in treatment, higher healthcare utilization and cost, less effective treatment and increased antibiotic resistance and Clostridioides difficile infection, demands a risk-based approach that simplifies the penicillin allergy delabeling process and establishes bridges with non-allergists. ${ }^{1,2}$

What have we learned that now makes the population level goal of penicillin delabeling achievable? First off, prevention is better than cure. We should critically examine pediatric populations for antibiotic use to address 
over-prescription of antibiotics including penicillins for viral infections. We should avoid labeling children with benign delayed exanthems that occur in the setting of a likely viral infection as penicillin allergic. When continued treatment is necessary we should in fact encourage "treating through" such reactions. When a label of penicillin allergy seems inevitable in a child we should address this label early and pay particular attention to antibiotic stewardship. New data on serum sickness-like reaction suggests that many of these are likely virally mediated and do not reproduce on ingestion challenge. ${ }^{3}$ Community based education programs can help disseminate timely information on penicillin allergy to dispel myths and alleviate fears. A label of penicillin allergy should be both viewed and approached as a threat to both individual and public health. On a public health level addressing penicillin allergy should be seen as a broad stewardship tool that provides a level of herd protectiveness against antibiotic resistance. On an individual level a label of penicillin allergy should be approached with the same routineness as any other preventative health check, and primary care physicians and providers should be trained to understand and manage low-risk penicillin allergy labels. ${ }^{4}$ Patients should regularly discuss their drug allergy passport with their healthcare providers such as pharmacists and physicians. Allergy passports should enable interoperability, high traceability and time-stamped information solving the problem of frequent unavailability and inaccuracy of drug allergy information. ${ }^{5}$ Risk stratification should occur and if in a low-risk category a patient should be given the option of direct oral challenge and delabeling. Risk stratification to identify by clinical history the low-risk penicillin allergic patients who would be appropriate for simple procedures is key. Several mechanisms now exist to risk stratify those labeled as penicillin allergic in routine clinical practice. These clinical prediction rules provide an evidence base to identify the majority of low-risk penicillin allergy labeled patients who are at low risk for rechallenge reactions. ${ }^{6,7}$ In current practice it is likely that less than $1 \%$ of such low-risk patients will be at risk for a reaction on ingestion challenge. ${ }^{1,8}$

To make widespread penicillin allergy delabeling an achievable and scalable goal we must be convinced of the safety of direct ingestion challenges. A randomized study allocated children 5 years or older with low-risk cutaneous reaction to penicillin skin testing followed by amoxicillin challenge versus 2 step direct oral challenge with amoxicillin with tolerance of amoxicillin of $96 \%$ of those with direct challenge and only minor reactions in the remainder. ${ }^{9}$ These results have recently been confirmed in an European population of children. ${ }^{10}$ Aside from the inconvenience and potential need for specialty assessment, for very low-risk patients, the use of skin testing would be expected to perform poorly considering their low pre-test probability of a reaction. Several other studies have demonstrated that a single or two-step direct ingestion challenge with penicillins such as amoxicillin is a safe and practical strategy to remove a label of penicillin allergy. ${ }^{11}$ Although there is evidence to support the use of risk stratification tools to delabel penicillin allergy under allergist guidance, we require an educational program on drug allergy for primary care physicians as well validation of these risk stratification tools, to show that low-risk penicillin delabeling can be achieved in this setting.

Even in the face of risk stratification and safety of direct ingestion challenge, populations are not equal in terms of their medical risk or antibiotic needs. Intuitively populations that serve to benefit from penicillins and other beta lactams have been shown to have inferior outcomes when labeled as penicillin allergic that would benefit from a delabeling intervention. This includes the association of penicillin allergy label and use of an alternative antibiotic with post-operative surgical site infections. ${ }^{12}$ Other settings where research has shown feasibility in delabeling include children in the emergency department, critically ill populations with high antibiotic needs, and pregnant women where the high rates of surgical delivery and group B Streptococcal colonization in pregnancy create a high demand for penicillin and cephalosporins as safe firstline drugs. ${ }^{1,13,14}$ Increasingly, assessment of unverified penicillin allergy has been recognized as an antibiotic stewardship intervention in immunocompromised states such as transplant and cancer where populations have much to gain by being delabeled. ${ }^{15}$

There is a "time for action" for removal of penicillin allergy labels on a population level but how do we achieve widespread implementation (Figure 1)? Policy changes should be driven by collaboration with Infectious diseases specialists and allergists who should join forces to pair antibiotic allergy management with antibiotic stewardship. In the community we need to educate parents and pediatricians to make them aware of the 
hazards of both unnecessary antibiotics and penicillin allergy labels for mild rashes that are often related to a viral infection and unlikely to recur. Primary healthcare providers should be given greater incentives to delabel penicillin allergic patients at the point-of-care and armed with decision support tools to facilitate risk stratification. For those whose history is not consistent with allergy this could include direct delabeling without testing. In the future, evidence may support that routine direct ingestion challenge with a penicillin and delabeling is safe in the primary care setting. Finally, by off-loading low-risk reactions to primary care providers we can then prioritize care of the patients with a higher-risk allergy and/or medical history by engagement with specialists who can provide more in-depth assessments and give them the best antibiotic options.

Figure 1: Addressing Beta-lactam Allergy: An Implementation Roadmap: There are currently many missed opportunities for community members and healthcare providers to take action forward on the "penicillin allergy delabeling" movement. This includes not only active measures to delabel patients by history and direct oral challenge and to identify high risk patients for prioritized penicillin allergy delabeling but also preventive measures to avoid unnecessary use and exposure to antibiotics and avoidance of unnecessary labeling in those with mild rashes of likely viral origin.

\section{References}

1. Castells M, Khan DA, Phillips EJ. Penicillin Allergy. N Engl J Med . Dec 12 2019;381(24):2338-2351. doi:10.1056/NEJMra1807761

2. Blumenthal KG, Phillips EJ. Positioning Drug Allergy Delabeling as a Critical Tool for Precision Medicine, Quality Improvement, and Public Health. J Allergy Clin Immunol Pract . Oct 2020;8(9):29162919. doi:10.1016/j.jaip.2020.07.046

3. Delli Colli L, Gabrielli S, Abrams EM, et al. Differentiating Between beta-Lactam-Induced Serum Sickness-Like Reactions and Viral Exanthem in Children Using a Graded Oral Challenge. J Allergy Clin Immunol Pract . Feb 2021;9(2):916-921. doi:10.1016/j.jaip.2020.08.047

4. Dona I, Caubet JC, Brockow K, et al. An EAACI task force report: recognising the potential of the primary care physician in the diagnosis and management of drug hypersensitivity. Clin Transl Allergy . 2018;8:16. doi:10.1186/s13601-018-0202-2

5. Brockow K, Aberer W, Atanaskovic-Markovic M, et al. Drug allergy passport and other documentation for patients with drug hypersensitivity - An ENDA/EAACI Drug Allergy Interest Group Position Paper.Allergy . Nov 2016;71(11):1533-1539. doi:10.1111/all.12929

6. Trubiano JA, Vogrin S, Chua KYL, et al. Development and Validation of a Penicillin Allergy Clinical Decision Rule. JAMA Intern Med . Mar 16 2020;doi:10.1001/jamainternmed.2020.0403

7. Plager J, Judd A, Blumenthal K. Role of clinical history in beta-lactam hypersensitivity. Curr Opin Allergy Clin Immunol. Aug 1 2021;21(4):320-326. doi:10.1097/ACI.0000000000000758

8. Romano A, Atanaskovic-Markovic M, Barbaud A, et al. Towards a more precise diagnosis of hypersensitivity to beta-lactams - an EAACI position paper. Allergy. Jun 2020;75(6):1300-1315. doi:10.1111/all.14122

9. Mustafa SS, Conn K, Ramsey A. Comparing Direct Challenge to Penicillin Skin Testing for the Outpatient Evaluation of Penicillin Allergy: A Randomized Controlled Trial. J Allergy Clin Immunol Pract . Sep - Oct 2019;7(7):2163-2170. doi:10.1016/j.jaip.2019.05.037

10. Prieto A, Munoz C, Bogas G, et al. Single-dose prolonged drug provocation test, without previous skin testing, is safe for diagnosing children with mild non-immediate reactions to beta-lactams.Allergy . Aug 2021;76(8):2544-2554. doi:10.1111/all.14800

11. Cooper L, Harbour J, Sneddon J, Seaton RA. Safety and efficacy of de-labelling penicillin allergy in adults using direct oral challenge: a systematic review. JAC Antimicrob Resist . Mar 2021;3(1):dlaa123. doi:10.1093/jacamr/dlaa123 
12. Boston BCDSP. Drug-induced anaphylaxis: a cooperative study.JAMA . 1973;224:613-615.

13. Staicu ML, Vyles D, Shenoy ES, et al. Penicillin Allergy Delabeling: A Multidisciplinary Opportunity. J Allergy Clin Immunol Pract . Oct 2020;8(9):2858-2868 e16. doi:10.1016/j.jaip.2020.04.059

14. Stone CA, Jr., Stollings JL, Lindsell CJ, et al. Risk-stratified Management to Remove Low-Risk Penicillin Allergy Labels in the ICU.Am J Respir Crit Care Med . Jun 15 2020;201(12):1572-1575. doi:10.1164/rccm.202001-0089LE

15. Trubiano JA, Thursky KA, Stewardson AJ, et al. Impact of an Integrated Antibiotic Allergy Testing Program on Antimicrobial Stewardship: A Multicenter Evaluation. Clin Infect Dis . Jul 1 2017;65(1):166174. doi:10.1093/cid/cix244

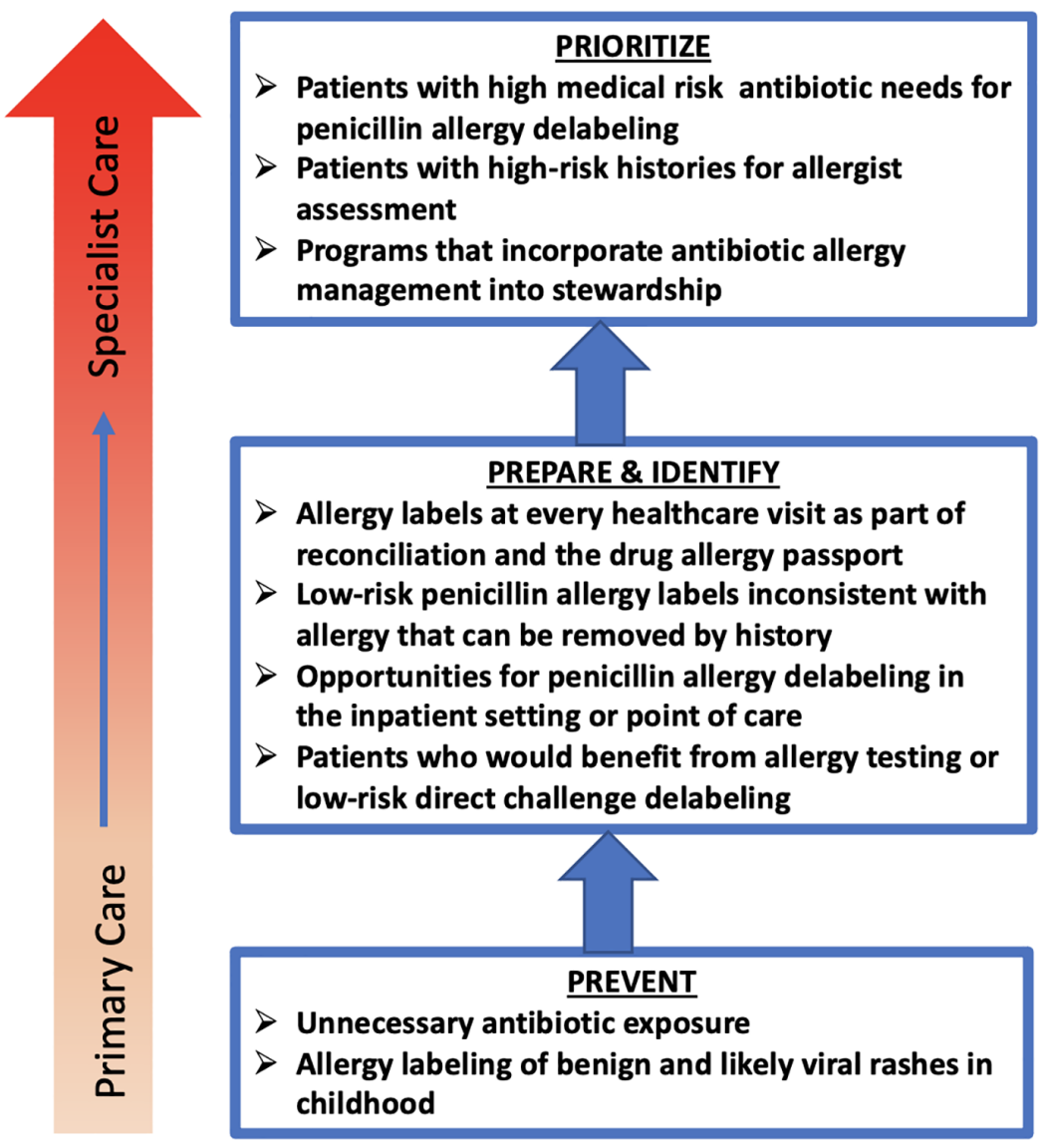

